\title{
Erweiterte Formen der roboterassistierten Lungenresektion
}

\author{
Joshua A. Scheinerman Jeffrey Jiang Stephanie H. Chang Travis C. Geraci Robert J. Cerfolio \\ Department of Cardiothoracic Surgery, New York University Langone Health, New York, NY, USA
}

\section{Schlüsselwörter}

roboterassistiert · Lobektomie · nicht kleinzelliges

Bronchialkarzinom - Manschettenresektion · Pneumonektomie .

adjuvante Radio(chemo)therapie

\section{Zusammenfassung}

Während der Lungenkrebs weiterhin die häufigste krebsbedingte Todesursache in den USA darstellt, bildet die Chirurgie in kurativer Intention weiterhin eine tragende Säule der Therapie. Seit mehr als einem Jahrzehnt kommen dabei im Rahmen der Lungenresektion bei nicht kleinzelligem Bronchialkarzinom (NSCLC) auch roboterassistierte Systeme zum Einsatz. Zu lokal begrenzteren Formen der Resektion wie z.B. Keilresektionen oder Lobektomien liegen umfangreiche Daten vor, die eine Reduktion der Krankenhausverweildauer und der postoperativen Schmerzen ebenso belegen wie verbesserte Raten der Lymphknotendissektion und des Auftretens von Komplikationen. Das roboterassistierte Vorgehen bietet eine Vielzahl technischer Vorzüge, z.B. bessere Optik, natürliche Handbewegungen für den Operateur bei der Steuerung der Instrumente sowie präzise Identifizierung der Gewebeebenen und entsprechend ergonomischer und sicherere Dissektion. Aufgrund dieser Vorteile wächst auch das Anwendungsspektrum für roboterassis- tierte Resektionen. In der vorliegenden Übersichtsarbeit werten wir die vorliegenden Daten zu erweiterten Formen der roboterassistierten Lungenresektion aus, insbesondere die Resektion nach Induktionstherapie, Manschetten-Lobektomie und Pneumonektomie. Darüber hinaus werden die Indikationen für diese komplexeren Resektionen betrachtet und die Daten und Outcomes aus der Erfahrung anderer Einrichtungen mit diesen Eingriffen wiedergegeben. Abschließend beschreiben wir die Strategie und Sichtweisen in unserer eigenen Einrichtung im Hinblick auf die genannten drei Formen der erweiterten Lungenresektion. Während die Anwendbarkeit und die Sicherheit der roboterassistierten Chirurgie bei diesen komplexen Lungenresektion noch Gegenstand kontroverser Debatten sind, hoffen wir durch Darstellung der vorliegenden Evidenz und Evaluierung der Wirksamkeit und Sicherheit für NSCLC-Patienten zur Klärung beizutragen.

(c) 2021 Scheinerman, Jiang, Chang, Geraci und Cerfolio

\section{Einleitung}

Knapp eine Viertelmillion Fälle von Lungenkrebs wurden 2017 allein in den USA diagnostiziert, und mehr als $80 \%$ davon waren nicht kleinzellige Bronchialkarzinome (non-small cell lung cancer, NSCLC;) [1] - das bedeutet einen substanziellen Bedarf an chirurgischen Resektionen zum Management dieser Erkrankungen. Parallel zu diesem steigenden Bedarf an Lungenresektionen

information@karger.com www.karger.com/kkp

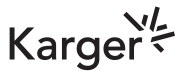

BOPEN ACCESS (c) 2021 The Author(s)

Published by S. Karger GmbH, Freiburg

This article is licensed under the Creative Commons Attribution 4.0 International License (CC BY) (http://www.karger.com/Services/Open AccessLicense). Usage, derivative works and distribution are permitted provided that proper credit is given to the au- sind signifikante Fortschritte in der thorakalen minimalinvasiven Chirurgie (MIC) erzielt worden, und so wurden im Zeitraum von 2002 bis 2018 knapp 57.000 MIC-Lobektomien durchgeführt, die meisten davon im letzteren Jahrzehnt [2]. Erheblich gestiegen ist in den letzten zehn Jahren auch der Anteil der Eingriffe, die mittels videoassistierter Thorakoskopie (VATS) oder als roboterassistierte Thoraxchirurgie durchgeführt werden. Und begleitend zum Fortschritt auf dem Gebiet der roboterassistierten Thorax- 
chirurgie wächst auch das Spektrum der Eingriffe, die mit einem robotischen System auf sichere Weise durchgeführt werden können. Komplexe Eingriffe mit einem hohen Maß an Adhäsionen und entsprechend erhöhtem Blutungsrisiko, z.B. Lungenresektionen nach Induktionstherapie, werden zunehmend häufig mit robotischen Systemen durchgeführt. Auch andere technisch anspruchsvolle Resektionen, z.B. Manschettenresektionen oder Pneumonektomien, sind mit einem robotischen System auf sichere Weise möglich. Die robotischen Systeme haben mehrere handfeste Vorteile - von einer niedrigeren Gesamt-Komplikationsrate über geringere Blutverluste, weniger postoperative Schmerzen und eine kürzere Krankenhausverweildauer bis hin zu einer verbesserten pathologischen Stadienbestimmung durch vollständigere Lymphknotenentfernung im Vergleich zur Thorakotomie [3]. In einer großen multizentrischen Studie zu 1336 roboterassistierten Lobektomien ermittelten Cerfolio et al. einen medianen Blutverlust von $50 \mathrm{ml}$, eine mittlere Operationsdauer von 136 min. und eine mediane Verweildauer von 3 Tagen [4], wobei alle Parameter sich im Verlauf der letzten 2 Jahre verbesserten (Veröffentlichung ausstehend). Angesichts der Vorteile bei der Resektion von NSCLC im Frühstadium ist ein vergleichbarer Nutzen der roboterassistierten Chirurgie auch bei den genannten erweiterten Resektionen wahrscheinlich.

\section{Roboterassistierte Lungenresektion nach Induktionsthe- rapie}

\section{Indikationen für die neoadjuvante Therapie}

Die ideale Behandlung des NSCLC im Frühstadium ist die vollständige chirurgische Resektion. Bei weiter fortgeschrittener Erkrankung wird das Management jedoch zunehmend komplex. Bei resezierbaren Tumoren ohne Beteiligung des Sulcus superior und ohne Befall der mediastinalen Lymphknoten besteht die Standardbehandlung in einer Operation, je nach endgültigem pathologischem Stadium eventuell in Verbindung mit einer adjuvanten systemischen Therapie und/oder Strahlentherapie. Negative pathologische Ränder vorausgesetzt, ist eine adjuvante Chemotherapie laut der aktuellen NCCN (National Comprehensive Cancer Network)-Leitlinie eine geeignete Option bei NSCLC im Stadium IB (Hochrisikopatienten), IIA und IIB. Bei Patienten mit N2 (mediastinalem Befall) hingegen umfasst die Standardtherapie eine neoadjuvante Chemotherapie oder Radiochemotherapie vor erneutem Staging und chirurgischer Resektion [5]. 1992 untersuchte das National Cancer Institute in einer Studie die Wirkung einer neoadjuvanten Chemotherapie beim NSCLC. Pass et al. teilten Patienten im Stadium IIIA (N2) per Randomisierung der Behandlung entweder mit Operation und nachfolgender Strahlentherapie oder mit neoadjuvantem Cisplatin/Etoposid und nachfolgender Operation zu. Von den 27 nachbeobachteten Patienten zeigte sich bei der neoadjuvant behandelten Gruppe mit 28,7 Monaten Überlebenszeit ein Trend zur Verlängerung des Überlebens im Vergleich zur anderen Gruppe mit 15,6 Monaten. Auch die Rezidivrate und krankheitsfreie Überlebenszeit fielen zugunsten der neoadjuvant behandelten Gruppe aus [6]. Unter- mauert werden diese Daten durch eine 2010 im Journal of Thoracic Oncology erschienene Metaanalyse von 13 randomisierten, kontrollierten Studien zur Behandlung von NSCLC-Patienten mit neoadjuvanter Therapie plus Operation im Vergleich zur alleinigen Operation. Diese Daten belegten für die Patienten mit NSCLC im Stadium III einen signifikanten Überlebensvorteil der neoadjuvanten Therapie gegenüber der Vergleichstherapie; die Subgruppenanalyse der mehr als 1500 Patienten ergab eine kombinierte HR von 0,84 bei $p=0,005$ [7] (Tab. 1).

\section{Chirurgische Resektion nach neoadjuvanter Therapie}

Eine Induktions-Chemotherapie beim NSCLC stellt den minimalinvasiven Thoraxchirurgen vor ganz besondere technische Herausforderungen. Dichte Adhäsionen und Fibrosen können im Kontext großer Tumoren mit entzündlicher Lymphadenopathie die Dissektion schwierig und gefährlich machen. Ein erster Bericht von 1994 zur Wirksamkeit der Induktions-Chemotherapie bei Patienten im Stadium IIIA ergab zwar keinen Unterschied in der postoperativen Sterblichkeit zwischen dem Arm mit neoadjuvanter Vorbehandlung und dem Arm ohne eine solche Vorbehandlung vor der Operation, doch in beiden Armen war eine hohe 30 -Tage-Mortalität von $7 \%$ zu verzeichnen [8]. Andere Daten hingegen belegen eine erhöhte postoperative 90-Tage-Mortalität und Gesamtkomplikationsrate. Roberts et al. verzeichneten bei neoadjuvanter Vorbehandlung erhöhte Raten an lebensbedrohlichen Komplikationen (26,5\% mit vs. $6,0 \%$ ohne neoadjuvante Vorbehandlung) und schwerwiegenden Komplikationen (47,1 vs. 19,4\%) sowie eine erhöhte 90-Tage-Mortalität (5,9 vs. 0\%) [9].

Einige Daten liegen zum Vergleich der minimalinvasiven mit der offenen Resektion bei NSCLC nach Induktionstherapie vor. Fang et al. stellten keine signifikanten Unterschiede bei der postoperativen Morbidität oder dem Gesamtüberleben in der VATS- gegenüber der Thorakotomiegruppe fest, allerdings war die VATS einerseits mit weniger postoperativen Schmerzen und andererseits mit einer geringeren Anzahl von Lymphknoten assoziiert [10]. Park et al. untersuchten retrospektiv 428 Patienten mit NSCLC im Stadium II-IIIA, die sich nach einer neoadjuvanten Chemooder Radiochemotherapie einer Lobektomie unterzogen hatten. Diese Gruppe wurde in zwei Kohorten unterteilt: Resektion mittels konventioneller Thorakotomie $(n=397)$ und mittels MIC ( $n=31$, davon 17 roboterassistiert, 14 VATS) [11]. Das 3-JahresGesamt- und krankheitsfreie Überleben waren bei beiden Gruppen vergleichbar, ebenso die Komplikationsrate. Die Verweildauer im Krankenhaus war in der MIC-Gruppe kürzer. Die Rate der R0-Resektionen war in beiden Gruppen vergleichbar; sie betrug 94\% in der Thorakotomie- und 97\% in der MIC-Gruppe. Ein Umstieg auf eine Thorakotomie erfolgte in 8/31 (26\%) der MIC-Fälle, begründet durch das Ausmaß der Erkrankung, schwere Adhäsionen sowie Blutungen [11].

Untersuchungen zu MIC-Resektionen bei neoadjuvantem NSCLC ergaben vergleichbar hohe Konversionsrate zur offenen Resektion. Huang et al. untersuchten 43 Patienten mit NSCLC im Stadium IIA-IIIB, die sich nach neoadjuvanter Behandlung einer VATS-Lungenresektion unterzogen. Bei den meisten Resektionen 
Tab. 1. Die Rolle der neoadjuvanten Chemotherapie bei fortgeschrittenem NSCLC

\begin{tabular}{|c|c|c|c|c|}
\hline Studie & Art der Studie & Datum & $N$ & Ergebnisse \\
\hline Pass et al. [6] & Monozentrische RKS & 1987-1992 & $\begin{array}{l}N=13 \text { neoadj. Chemo + OP; } 14 \\
\text { OP + RT }\end{array}$ & $\begin{array}{l}\text { Beide Gruppen 0\% operative Mortalität } \\
\text { Überleben 28,7 vs. 15,6 Monate (neoadj. vs. OP); Trend } \\
\text { zugunsten von neoadj. DFS 12,7 vs. 5,8 Monate (neoadj. vs. } \\
\text { OP); Trend zugunsten von neoadj. Rezidivierung } 11 / 12 \text { OP } \\
\text { vs. } 8 / 11 \text { neoadj. Raten vollständiger Tumor- und LK-Resek- } \\
\text { tion vergleichbar }\end{array}$ \\
\hline Song et al. [7] & Metaanalyse, retrosp. & $1985-2004$ & $\begin{array}{l}N=1.637 \text { neoadj. }+ \text { OP; } 1587 \\
\text { nur OP }\end{array}$ & $\begin{array}{l}\text { Überleben verbessert im neoadj. Arm; HR }=0,84 ; p<0,005 \text {, } \\
\text { bei heterog. Pop. und bei Pat. mit NSCLC Stadium III }\end{array}$ \\
\hline Rosell et al. [8] & Prosp. RCT, & 1989-1991 & $\begin{array}{l}N=30 \text { neoadj. }+\mathrm{OP}+\mathrm{RT} ; 30 \\
\text { nur OP + RT }\end{array}$ & $\begin{array}{l}\text { Medianes Überleben } 26 \text { vs. } 8 \text { Monate, neoadj. vs. nur OP } \\
\text { DFS } 20 \text { vs. } 5 \text { Monate, neoadj. vs. nur OP } \\
\text { Rezidivrate } 56 \text { vs. } 74 \% \text {, neoadj. vs. nur OP }\end{array}$ \\
\hline Roberts et al. [9] & Retrosp. Kohorte & 1997-1999 & $N=34$ neoadj., 67 nur OP & $\begin{array}{l}\text { Erhöhte Inzidenz lebensbedrohlicher postop. Komplika- } \\
\text { tionen in der neoadj. Gruppe; schwere Komplikationen 47,1 } \\
\text { vs. } 19,1 \% \text {; } \\
\text { erhöhte Tracheotomie-Rate in der neoadj. Gruppe } \\
2 \text { Todesfälle innerhalb von } 90 \text { Tagen in der neoadj. Gruppe, } \\
0 \text { in der nur-OP-Gruppe }\end{array}$ \\
\hline
\end{tabular}

handelte es sich um Lobektomien, es waren jedoch auch 9 Bronchial-Manschetten-Lobektomien, 5 Bilobektomien und 4 Pneumonektomien darunter. Die Überlebensrate für die gesamte Kohorte betrug nach 1, 2 und 3 Jahren 94, 79 bzw. 65\%. Bei 40 Patienten wurde eine R0-Resektion erreicht. Die Mortalität lag bei 2,3\% (1/43 Patienten), und 5 Patienten entwickelten postoperative Komplikationen. Während bei keinem Patienten eine Umstellung auf «konventionelle Thorakotomie» erfolgte, wurde in 7 (16,7\%) der Fälle eine «hybride VATS» durchgeführt; der Grund hierfür war die Nähe des Tumors zu großen Gefäßen und/oder Bronchien im Kontext einer mittelschweren bis schweren Entzündung und Fibrose infolge der neoadjuvanten Therapie [12]. In einer retrospektiven multizentrischen Untersuchung analysierten Cerfolio et al. 223 Patienten mit okkulter oder evidenter N2-Erkrankung, die sich einer roboterassistierten Resektion unterzogen hatten. $15,2 \%$ von ihnen erhielten eine neoadjuvante Therapie, und 8 Patienten erhielten außerdem eine präoperative Strahlentherapie. Schwerwiegende Komplikationen waren mit 10,1\% in der Gruppe mit neoadjuvanter Vorbehandlung vergleichbar häufig wie in der Gruppe ohne eine solche Vorbehandlung vor der Operation. Die R0-Resektionsrate betrug in der neoadjuvanten Gruppe $98,1 \%$ bei einer 30 - und 90 -Tages-Mortalität von $0 \%$. Bei $15 \%$ der neoadjuvanten Patienten wurde die Resektion auf eine Thorakotomie umgestellt. Keine dieser Umstellungen erfolgte aufgrund von Blutungen, sondern in der Regel zur Tumorresektion nach Entdeckung eines ausgedehnten Befalls der mediastinalen Lymphknoten [13] (Tab. 2).

Eine Operation nach einer Induktions-Immuntherapie ist mit ähnlichen Risiken verbunden. Broderick et al. untersuchten die Outcomes von 20 Patienten mit NSCLC im Stadium I-IIIA, bei denen nach neoadjuvanter Gabe von Nivolumab eine Lungenresektion durchgeführt wurde - in 13 Fällen initial mittels VATS oder roboterassistiert. Die perioperative Mortalität betrug $0 \%$ und die Morbidität 50\%, wobei es sich meist um Vorhofflimmern handelte. Bei drei Viertel der Patienten wurde eine Lobektomie vorgenommen, bei weiteren $10 \%$ eine Pneumonektomie. Von den 13 Eingriffen, die zunächst minimalinvasiv versucht wurden, wurden 7 (54\%) auf eine Thorakotomie umgestellt. Diese Konversionen erfolgten zum Teil aufgrund von Blutungen und/oder dichten Adhäsionen im Brustraum. Die übrigen Konversionen waren durch stark entzündete mediastinale und hiläre Lymphknotenstationen begründet [14].

Aktuelle Daten belegen, dass eine Induktionsbehandlung mit Chemo-, Strahlen- oder Immuntherapie mit vermehrtem Umstellungsbedarf von einer minimalinvasiven auf eine offene Resektion einhergehen kann. Dieselben Studien belegen jedoch auch, dass die minimalinvasive Lungenresektion nach einer Induktionstherapie $\mathrm{zu}$ vergleichbaren Gesamtüberlebensraten führt wie die offene Resektion. Nach Auswahl der richtigen Patienten mit lokal fortgeschrittener Erkrankung ist die roboterassistierte Resektion nach vorausgehender Induktionstherapie somit sicher, wirksam und in Bezug auf Überleben und Komplikationen vergleichbar mit der konventionellen, offenen Resektion.

Roboterassistierte Techniken für die Operation nach neoadjuvanter Behandlung

Roboterassistierte Lungenresektionen werden in unserer Einrichtung mit dem Operationssystem Da Vinci Xi (Intuitive Surgical Inc., Mountain View, CA, USA) durchgeführt. Mit einer Bronchoskopie wird die korrekte Platzierung des doppellumigen Schlauchs sichergestellt, und der Patient wird in Seitenlage gebracht. Wir verwenden als standardisiertes Setup einen vierarmigen Zugang und ein $0^{\circ}$-Endoskop mit kongruenter Portanlage im 8. Interkostalraum und mit einem Assistenzport für den Assistenten am Patienten, der auch als Luftdichtung bei der $\mathrm{CO}_{2}$-Insufflation der Pleurahöhle genutzt wird [16]. In der Regel wird der am weitesten anterior gelegene Port (ein 12-mm-Port) eine Rippe höher angelegt als die anderen drei Roboterports, um eine Verlet- 
Tab. 2. Outcomes minimalinvasiver vs. offener Resektionen nach neoadjuvanter Therapie bei NSCLC

\begin{tabular}{|c|c|c|c|c|}
\hline Studie & Art der Studie & Datum & $N$ & Ergebnisse \\
\hline Fang et al. [10] & $\begin{array}{l}\text { Retrosp. monoz. } \\
\text { Kohorte }\end{array}$ & 2013-2017 & $N=67$ offen, 14 VATS & $\begin{array}{l}\text { Weniger postop. Schmerzen, geringere Anzahl entnommener LK } \\
\text { in der VATS-Gruppe }\end{array}$ \\
\hline Park et al. [11] & $\begin{array}{l}\text { Retrosp. monoz. } \\
\text { Kohorte }\end{array}$ & $2002-2013$ & $\begin{array}{l}N=397 \text { offen, } 31 \text { MIC (17 } \\
\text { roboterassistiert, 14 VATS) }\end{array}$ & $\begin{array}{l}\text { R0-Resektionsrate und postop. Morbidität vergleichbar } \\
\text { Kürzere WWD in der MIC-Gruppe ( } 4 \text { vs. } 5 \text { Tage) } \\
\text { DFS und OS vergleichbar; DFS } 49 \text { vs. } 42 \% \text {, MIC vs. offen } \\
26 \% \text { Konversion zu offener OP in der MIC-Gruppe }\end{array}$ \\
\hline Huang et al. [12] & $\begin{array}{l}\text { Retrosp. monoz. } \\
\text { Kohorte }\end{array}$ & 2006-2012 & $N=43$ & $\begin{array}{l}28 \text { Lobektomien, } 9 \text { Manschettenres., } 5 \text { Bilobektomien, } 4 \text { Pneumo- } \\
\text { nektomien } \\
\text { 16,7\% Konversion zu «Hybrid-VATS», Rest VATS } \\
\text { Medianer Blutverlust } 253 \text { ml; OP-Dauer } 160 \text { Min; mittlere Dauer } \\
\text { KH-Aufenthalt 5,45 Tage } \\
\text { Inzidenz postop. Komplikationen 11,9\% } \\
1 \text { perioperativer Todesfall; 3-Jahres-Überleben 65\% }\end{array}$ \\
\hline Bott et al. [14] & \multicolumn{2}{|c|}{$\begin{array}{l}\text { Multizentr., einarmige 2015-2016 } \\
\text { Kohorte }\end{array}$} & $N=20$ & $\begin{array}{l}15 \text { Lobektomien, } 2 \text { Pneumonektomien, } 1 \text { Manschettenres., } 1 \\
\text { Bilobektomie } \\
13 \text { versuchte VATS/RATS, 54\% Konversion zu offener OP } \\
\text { Mediane OP-Dauer } 228 \text { Min., WWD } 4 \text { Tage, keine periop. Mor- } \\
\text { talität; 50\% postop. Morbidität }\end{array}$ \\
\hline Cerfolio et al. [15] & Retrosp., multiz. & 2007-2016 & $N=223 ; 34$ mit neoadj. & $\begin{array}{l}\text { Alle } 34 \text { mit R0-Resektion; 15\% Konversionsrate (nicht blutungs- } \\
\text { bedingt), 12\% mit schweren postop. Komplikationen } \\
\text { 3-Jahres-Überleben in der neoadj. Gruppe 60,1\% } \\
\text { OS deutlich besser bei Pat. mit <2 pos. LK }\end{array}$ \\
\hline
\end{tabular}

zung oder Betäubung des Nervs zum oberen Musculus rectus abdominis zu vermeiden. Vor dem Andocken wird eine Interkostalnervenblockade über mehrere Ebenen durchgeführt.

Die Dissektion beginnt in der Regel mit einer sorgfältigen Exploration der Brusthöhle um sicherzustellen, dass weder Pleurametastasen noch unvermutete freie Flüssigkeit vorhanden sind. Das untere Ligamentum pulmonale wird durchtrennt und die Lunge mit dem 4. Arm nach superior retrahiert, und die Lymphknoten von Station 9 und anschließend 8 werden entnommen. Danach wird das subcarinale Lymphknotenpaket vollständig entfernt, ebenso die gesamten Stationen 2-4 auf der rechten Seite bzw. 5-6 auf der linken. Bei einer Resektion nach Induktionstherapie prüfen wir sorgfältig, welcher Aspekt der Dissektion am stärksten durch Adhäsionen erschwert wird. Die Lokalisation des Tumors, Lymphadenopathie und Bereiche früheren positiven Nodalbefalls werden in Bezug auf die hilären Gefäße und den Bronchus beurteilt. Wir beginnen vorzugsweise mit einer posterioren Dissektion. Auf der linken Seite erlaubt die posteriore Ansicht die Identifikation der PA sowie der PA-Äste A2 und A6, um die posteriore Fissur vollständig öffnen zu können. Auf der rechten Seite kann man durch den posterioren Zugang den rechten Oberlappenbronchus umschlingen und durchtrennen, bevor PA-Äste ligiert werden. Mit diesen Tricks erhöht man die Sicherheit der PA-Dissektion. Vor der Dissektion dieser Bereiche erfolgt in ausgewählten Fällen eine hiläre Kontrolle durch sorgfältige Dissektion um die Pulmonalvenen stumpf und mit bipolarer Energie sowie durch Dissektion der proximalen Pulmonalarterie. Wenn der Tumor recht weit proximal lokalisiert ist, werden mit intraperikardialer Technik die Pulmonalarterie und die Pulmonalvenen umschlungen. Nachdem diese Kontrolle erreicht ist, wird die Dissektion durchgeführt. Im Fall einer starken Blutungen aus der Pulmonalarterie oder -vene kann der Operateur das Hilum wirksam verschließen. Während der Dissektion im Umfeld von Gefäßstrukturen liegen mehrere Schwämme in der Brust bereit, um bei Bedarf Druck ausüben zu können. Ein Thorakotomie-Sieb sollte sich im Raum befinden, und Blutprodukte sollten bereitgehalten werden.

Unsere Arbeitsgruppe hat bereits früher über das Vorgehen und die Denkweise des Operateurs in der roboterassistierten Thoraxchirurgie geschrieben, zum Aspekt der Umstellung auf eine offene Operation bei massiven Blutungen. Das Paradigma der $7 \mathrm{P}$ «preparation, pressure, patience, poise, products, partner, prolene» (Vorbereitung, Druckausübung, Geduld, Ruhe, Produkte, Partner und Prolen) gilt auch hier, zuzüglich der vorab hergestellten hilären Kontrolle [15]. Dies sind unserer Einschätzung nach die wichtigsten Punkte bei der Durchführung einer roboterassistierten Lungenresektion nach Induktionstherapie, und von besonders kritischer Bedeutung ist hierbei das Potenzial für massive Blutungen der großen Gefäße und die deshalb erforderliche prophylaktische Kontrolle der Hilusgefäße vorab.

\section{Roboterassistierte Manschettenresektion}

\section{Indikationen für die Manschettenresektion}

Ein zentral lokalisierter NSCLC-Tumor stellt eine besondere Herausforderung für die Erreichung einer R0-Resektion dar. Eine Beteiligung der Atemwege einschließlich des proximalen Lappenbronchus oder Hauptstamms verunmöglicht eine kleinere anatomische Resektion und hat in der Vergangenheit eine Pneumonektomie erforderlich gemacht. Die Manschettenresektion 
Tab. 3. Vergleich der Outcomes von Manschetten-Lobektomie und Pneumonektomie

\begin{tabular}{|c|c|c|c|c|}
\hline Studie & Art der Studie & Datum & N & Ergebnisse \\
\hline Deslauriers et al. [19] & Monoz. Kohorte & $1972-2000$ & $\begin{array}{l}184 \text { Mansch. } \\
1046 \text { PN }\end{array}$ & $\begin{array}{l}\text { OP-Mortalität 1,3 (Mansch.) vs. 5,3\% (PN) } \\
\text { 5-J-Überleben } 52 \text { (Mansch.) vs. 31\% (PN) } \\
\text { 5-J-Überleben bei Stadium I/II } 63 \text { (Mansch.) vs. 43\% (PN) } \\
\text { KEIN Überlebens-Unterschied bei Stadium III } \\
\text { Lokoregionäre Rezidive 22\% (Mansch.) vs. 35\% (PN) }\end{array}$ \\
\hline Okada et al. [20] & Monoz. Kohorte & 1984-1998 & $\begin{array}{l}151 \text { Mansch. } \\
60 \text { Mansch. parallelisiert } \\
60 \text { PN }\end{array}$ & $\begin{array}{l}\text { OP-Mortalität } 0 \text { (Mansch.) vs. 2\% (PN) } \\
\text { Morbidität } 13 \text { (Mansch.) vs. 22\% (PN) } \\
\text { Rezidive 8\% (Mansch.) vs. 10\% (PN) } \\
\text { 5-Jahres-Überleben } 48 \text { (Mansch.) vs. 28\% (PN) }\end{array}$ \\
\hline Pages et al. [18] & Landesweite Kohorte & $2005-2014$ & $\begin{array}{l}941 \text { Mansch. } \\
5318 \text { PN }\end{array}$ & $\begin{array}{l}\text { OP-Mortalität } 5 \text { (Mansch.) vs. 5,89\% (PN) } \\
\text { HR Langzeitüberleben PN vs. Mansch. 1,63 (parallelisierte } \\
\text { Daten; } p=0,002 \text { ) } \\
\text { 5-J-DFS 71,8 (Mansch.) vs. } 60,76 \% \text { (PN) }\end{array}$ \\
\hline
\end{tabular}

der Atemwege wurde als Alternative zur Pneumonektomie entwickelt. Sie wurde ursprünglich nur bei sehr gebrechlichen Patienten mit geringer pulmonaler Reserve in Betracht gezogen, da man eine mangelhafte Tumorresektion mit knappen Rändern befürchtete. In jüngerer Vergangenheit hat die Manschettenresektion als primäre chirurgische Option an Akzeptanz gewonnen, und zwar auch für Patienten, die eine Pneumonektomie tolerieren würden [16]. Derzeit umfasst die Indikation der Manschettenresektion sämtliche zentral lokalisierten Tumoren im Stadium I und II, bei denen die Manschettenresektion technisch durchführbar ist $[17,18]$.

Den Nutzen der Manschettenresektion bei zentralen Tumoren im Frühstadium veranschaulicht eine große retrospektiven Fallserie eines einzelnen Zentrums in Frankreich, welche 1230 Patienten mit 184 Manschettenresektionen umfasst. Die Autoren belegen ein signifikant verbessertes 5-Jahres-Überleben (52 vs. 31\%) und eine niedrige lokoregionale Rezidivrate (22 vs. 35\%) für die Manschettenresektion im Vergleich zur Pneumonektomie bei Patienten im Stadium I/II [19]. Ebenso berichteten Okada et al. bei 120 parallelisierten Patienten - 60 Manschettenresektionsn vs. 60 Pneumonektomien - über bessere Ergebnisse einschließlich einer 5-Jahres-Überlebensrate von 48 vs. 28\% [20]. Nationale Daten aus Frankreich zum Vergleich von 941 Manschetten-Lobektomien mit 5.318 Pneumonektomien führten zu der Empfehlung, eine Manschetten-Lobektomie bei Krebs im Frühstadium wann immer möglich durchzuführen, basierend auf einer vergleichbaren Langzeitmortalität und möglicherweise besseren Ergebnissen bei Parallelisierung nach Propensity-Score (18) (Tab. 3).

Patienten mit Krankheitsbeteiligung des proximalen Anteils der Pulmonalarterie stellen insofern einen Sonderfall dar, als sie nachweislich von einer pulmonalen Arterioplastik mit oder ohne bronchiale Manschettenresektion profitieren. Defekte der Arterie können unterschiedlich ausgeprägt sein, und als Rekonstruktionstechnik wurden Patch-Rekonstruktion, primäre Anastomose und Interpositionsplastik beschrieben [21]. Rendina et al. haben über 20 Jahre Erfahrung mit pulmonaler Arterioplastik im Rahmen einer Lobektomie bei 105 Patienten berichtet. Ihre Serie um- fasst 47 Pulmonalarterien-Manschetten und 65 PA-Rekonstruktionen in Verbindung mit Bronchialmanschetten. Die operative Mortalität betrug hier $0,95 \%$ und die 5-Jahres-Überlebensrate $44 \%$. Zuvor befürchtete Komplikationen wie PA-Thrombose oder Blutungen waren selten; ein einziger Fall von Thrombose trat auf. Das langfristige krankheitsfreie Überleben entsprach dem, das man bei kleineren Resektionen im jeweiligen Stadium erwarten würde [22, 23]. Eine Metaanalyse zur Manschetten-Lobektomie mit pulmonaler Arterioplastik ergab außerdem vergleichbare operative Risiken, Komplikationsraten und Langzeitmortalität wie nach isolierter Manschetten-Lobektomie sowie einen Trend zu besseren Outcomes als nach Pneumonektomie [24].

\section{Minimalinvasive Manschettenresektionen}

Die Vorteile der minimalinvasiven Chirurgie sind umfassend dokumentiert [25]. Wie weiter oben bereits dargelegt hat die Einführung der roboterassistierten Chirurgie zusätzlich zur videoassistierten Chirurgie weitere Verbesserungen bei Komplikationsraten, Verweildauern und 30-Tage-Mortalität bewirkt [26]. Manschettenresektionen waren bis dahin auf offene Eingriffe beschränkt gewesen - von einigen wenigen Spezialzentren abgesehen -, da die VATS durch begrenzte Manövrierfähigkeit und mangelnde Tiefenwahrnehmung gekennzeichnet ist. Roboterassistierte Bronchoplastiken wurden zunächst an Kadavermodellen beschrieben, bevor sie als VATS/RATS-Hybridtechnik zur Anwendung kamen [27]. Die Herausforderungen der Bronchoplastik passen gut zur Roboterplattform, die präzises intrathorakales Nähen ermöglicht.

Die Verbreitung roboterassistierter Systeme hat in den letzten zehn Jahren zugenommen. Doch nur wenige Einrichtungen haben Fallserien zu ihren Ergebnissen publiziert. Von Jiao et al. stammt die größte Fallserie mit 67 Patienten und einem breiten Spektrum von Indikationen für die Manschetten-Lobektomie, einschließlich solcher mit Metastasenbefall peribronchialer Lymphknoten. Die Arbeit belegt die Sicherheit und Durchführbarkeit des Verfahrens mit einer 90-Tage-Mortalität von Null, wenigen Komplikationen und keinen Umstellungen auf einen offe- 
Tab. 4. Outcomes roboterassistierter Manschettenresektionen

\begin{tabular}{|c|c|c|c|c|}
\hline Studie & Art der Studie & Datum & $N$ & Ergebnisse \\
\hline Jiao et al. [28] & Serie & 2014-2018 & 67 & $\begin{array}{l}\text { 90-Tage-Mortalität 0\% } \\
\text { 0\% Konversion zur offenen OP } \\
\text { Komplikationsrate 13\% (1 bronchiale Stenose, } 2 \text { anhaltende Luftleckage, } \\
1 \text { Pneumonie, } 1 \text { Chylothorax, 1 CVA) } \\
\text { Mittlere OP-Dauer 166,5 Min. } \\
\text { Durchschnittl. WWD 6,8 Tage }\end{array}$ \\
\hline Geraci et al. [16] & Serie & 2010-2019 & 23 & $\begin{array}{l}\text { 90-Tage-Mortalität 0\% } \\
\text { R0-Resektionsrate 100\% } \\
1 \text { Konversion zu offener OP wegen Anastomosenspannung } \\
\text { Morbidität 9\% (1 Pneumonie, } 1 \text { Transfusion) } \\
\text { Rezidivrate 0\%; mediane Nachbeobachtungsdauer } 18 \text { Monate } \\
\text { Mediane OP-Dauer } 205 \text { Min. } \\
\text { Mediane WWD } 3 \text { Tage }\end{array}$ \\
\hline Pan et al. [30] & Serie & 2014-2015 & 21 & $\begin{array}{l}\text { 30-Tage-Mortalität 4,8\% } \\
\text { R0-Resektionsrate 90,5\% } \\
\text { 4,8\% Konversion zur offenen OP wegen verkalkter Lymphknoten } \\
\text { Komplikationsrate 19\% (2 Pneumonie, } 2 \text { Pyothorax, } 1 \text { Anastomose-Leckage) } \\
\text { Mittlere OP-Dauer 158 Min. } \\
\text { Durchschnittl. WWD 10,7 Tage }\end{array}$ \\
\hline Qiu et al. [32] & Retrosp. Kohorte & $2012-2017$ & $\begin{array}{l}188 \text { Mansch., } \\
49 \text { roboterass., } \\
73 \text { VATS, } 66 \text { offen }\end{array}$ & $\begin{array}{l}\text { Gesch. Blutverl. } 119 \text { (R) vs. } 182 \text { (VATS) vs. } 222 \text { (offen) } \\
\text { OP-Dauer } 200 \text { (R) vs. } 291 \text { (VATS) vs. } 240 \text { (offen) } \\
\text { WWD 7,7 (R) vs. 10,2 (VATS) vs. 10,2 (offen) } \\
\text { Schlauchdrainage-Tage 4,2 (R) vs. 7,2 (VATS) vs. 6,9 (offen) } \\
\text { Negative Ränder } 100 \text { (R) vs. 91,2 (VATS) vs. 98,3\% (offen) } \\
\text { Keine Unterschiede bei Mortalität oder Morbidität vor oder nach Propensity } \\
\text { Matching }\end{array}$ \\
\hline
\end{tabular}

nen Eingriff. Zu den schwerwiegenden Komplikationen zählten eine Anastomosenstriktur, ein Chylothorax, ein Schlaganfall und eine erneute stationäre Aufnahme wegen Pneumonie [28]. Unsere Gruppe hat die zweitgrößte Fallserie vorgelegt; sie umfasst 23 Patienten ohne kurzfristige Mortalität und mit minimaler Morbidität in Form eines Umstiegs auf eine offene Operation wegen Besorgnis erregender Anastomosenspannung, einer erneuten stationären Behandlung wegen Pneumonie sowie einer Transfusion wegen asymptomatischer Anämie. Über eine mediane Nachbeobachtungszeit von 18 Monaten trat bei keinem der Patienten ein lokoregionales Rezidiv auf [29]. Pan et al. hatten zuvor über eine vergleichbare Serie von 21 roboterassistierten Manschettenresektionen mit ähnlichen Ergebnissen berichtet, allerdings mit einem Todesfall innerhalb von 30 Tagen und geringfügig höheren Komplikationsraten [30]. Eine aktuelle retrospektive Übersichtsarbeit $\mathrm{zu}$ roboterassistierten Manschettenresektionen im Vergleich zu VATS- und offenen Eingriffen ergibt mindestens gleichwertige Kurzzeitergebnisse bei gewichtet-parallelisierten Patienten und deutet auf weniger Blutungen, kürzere Verweildauern und kürzere Operationszeiten hin [31] (Tab. 4). Während die kurzfristigen onkologischen Ergebnisse vielversprechend aussehen, gilt es nun die langfristigen Daten abzuwarten.

\section{Technik der roboterassistierten Manschettenresektion}

Unser Vorgehen bei der roboterassistierten Manschetten-Lobektomie umfasst einen komplett portalen Roboter-Zugang mit dem Da Vinci Xi System (Intuitive Surgical Inc, Mountain View, CA,
USA) und einer $0^{\circ}$-Kamera. Vor der Portanlage werden mittels Bronchoskopie die Tumorausdehnung und die endobronchiale Beteiligung ermittelt. Vier Ports für die Roboterarme plus ein Assistenzport werden in Lobektomie-Standardpositionen angelegt wie weiter oben beschrieben.

Im Folgenden wird eine Lobektomie des rechten Oberlappens beschrieben, da dies die am häufigsten durchgeführte konkomitante Lobektomie ist. Eine mediastinale Lymphadenektomie wird durchgeführt, wobei sorgfältig darauf geachtet wird, das gesamte subcarinale Lymphknotenpaket zu entfernen, um so den Abgang des rechten und linken Hauptbronchus zu visualisieren. Das anatomische Dreieck um den Abgang des rechten Oberlappenbronchus herum wird unter Resektion des zugehörigen Lymphknoten-Levels 10 sauber präpariert. Die Fissur wird dann entweder, wenn sie vollständig ist, bipolar freipräpariert oder getunnelt und das Parenchym geklammert, um die durchgehende Arterie freizulegen. Die posterior aufsteigende Arterie und die Vena azygos werden geklammert, um eine vollständige Mobilisierung der Trachea zu ermöglichen und eine Fistel zur Bronchusanastomose zu verhindern. Die $\mathrm{F}_{\mathrm{i}} \mathrm{O}_{2}$ wird auf $21 \%$ verringert, und der ROL-Bronchus wird mit einer unipolaren Schere durchtrennt. Nach Durchtrennung des Truncus anterior und der posterioren Fissur wird die restliche Lobektomie durchgeführt. Ein Video zu diesem Verfahren finden Sie unter https://www.youtube.com/watch?v = 0_k3Sgro5E.

Bei Läsionen, die eine Rekonstruktion der Pulmonalarterie erfordern, erfolgen proximale und distale Kontrolle mittels Gefäß- 
schlingen und flexibler Gefäßklemme (Cygnet; Peters Surgical, Bobigny, Frankreich), welche durch eine separate Inzision eingebracht und an die Brustwand angepasst wird, oder mittels einer sicheren Bulldog-Klemme (Scanlan, Saint Paul, MN, USA) durch die Roboter-Ports. Die distale Kontrolle wird in ähnlicher Weise oder durch Abklemmen der Pulmonalvene hergestellt. Heparin wird nicht routinemäßig verabreicht, außer bei verlängerter Abklemmzeit. Die Bronchotomie erfolgt unter Infrarot-Visualisierung und Bronchoskopie (Firefly; Intuitive Surgical, Sunnyvale, CA, USA). Hierbei wird mit der proximalen Bronchotomie begonnen, um die distale Ausdehnung der Läsion zu visualisieren. Ein Gefrierschnitt des proximalen und distalen Bronchus wird vorgenommen; im Fall positiver Ränder erfolgt eine weitere Resektion.

Für die Bronchusanastomose wird der Atemweg so ausgerichtet, dass der membranöse Anteil parallel zur Wirbelsäule verläuft, und zwei fortlaufende Stratafix-Nähte (Johnson \& Johnson, New Brunswick, NJ, USA) werden gesetzt. Zuerst wird der knorpelige Anteil der Anastomose von 6 bis 12 Uhr am proximalen Atemweg (Übergang zu Übergang zwischen knorpeligen und membranösen Anteilen) fertiggestellt (Video unter https:// www.youtube.com/watch?v = HVnBIeAgXdU), bevor die Lunge nach superior und anterior retrahiert wird, um eine zweite Naht am membranösen Anteil zu setzen. Die Pneumostase wird durch erneute Insufflation unter Kochsalzlösung geprüft, und wenn ein Luftleck vorliegt, wird eine Einzelnaht zu Reparatur gesetzt. Eine Abstützung erfolgt routinemäßig nicht, außer wenn der Patient zuvor einer neoadjuvanten Therapie unterzogen wurde [29].

\section{Roboterassistierte Pneumonektomie}

\section{Indikationen für die Pneumonektomie}

Die Pneumonektomie ist angesichts der mit ihr assoziierten Morbidität und Mortalität nach wie vor ein Eingriff der letzten Wahl [29]. Unter den elektiven Lungenresektionen ist sie die mit dem höchsten Risiko behaftete; die operative Mortalität liegt bei 3 bis $12 \%$, während in den aktuellen Leitlinien ein Risiko von $7 \%$ oder weniger als akzeptabel bezeichnet wird [33]. Das Sterberisiko steigt zusätzlich bei Vorliegen einer entzündlichen Erkrankung und vorheriger Induktionstherapie; hier werden Raten von über $12 \%$ berichtet [34]. Auch die Rate kardiovaskulärer Komplikationen ist signifikant höher als bei anderen, kleineren Resektionen; sie wird mit 20 bis $60 \%$ beziffert, und viele dieser Komplikationen sind lebensbedrohlich und machen eine sofortige Intervention erforderlich [35]. Weitere Morbiditäten sind Pneumonie, bronchopleurale Fistel, Lähmung des Nervus laryngeus recurrens, Lungenembolie und verzögerte Extubation [36, 37]. Bei zentral lokalisiertem NSCLC mit Beteiligung hilärer Strukturen durch direkte Tumorinvasion oder extensive Erkrankung der hilären Lymphknoten ist für eine R0-Resektion eine Pneumonektomie erforderlich. Weitere Indikationen umfassen eine multilobäre Erkrankung oder metachrone Läsionen in anderen Lappen. Darüber hinaus bleibt eine Pneumonektomie als Reserveoption, wenn sich eine lungenerhaltende Resektion als onkologisch unzureichend erweist und die pulmonale Reserve des Patienten ausreichend ist [29].

\section{Minimalinvasive Pneumonektomie}

Wie bereits dargelegt, sind die Vorteile der MIC validiert und umfassen einen geringeren Blutverlust, kürzere Verweildauer, weniger postoperative Schmerzen und gleichwertige onkologische Ergebnisse. Die Verbreitung der VATS- oder roboterassistierten Pneumonektomie nimmt jedoch nur langsam zu und wird durch technische, sicherheitsbezogene und onkologische Bedenken gebremst. Dadurch liegen bisher nur wenige Fallserien zu VATSPneumonektomien und lediglich Fallberichte über roboterassistierte Operationen vor.

Thorakoskopische Pneumonektomien sind bisher in drei Fallserien beschrieben worden, alle mit ungefähr ähnlichen Ergebnissen. Sie alle gelangen im Prinzip zu dem Schluss, dass die VATSPneumonektomie ein durchführbares und sicheres Verfahren mit äquivalenten onkologischen Ergebnissen, wenngleich mit hoher Konversionsrate, darstellt. Battoo et al. untersuchten an einem einzelnen Zentrum 107 Patienten (davon 67 VATS) mit vergleichbaren postoperativen Komplikationsraten, keinen größeren intraoperativen Blutungen, einer Konversionsrate von $16 \%$ und verminderten Schmerzen nach 1 Jahr [38]. Liu et al. verglichen 64 offene Eingriffe mit 32 parallelisierten VATS-Pneumonektomien und stellten ähnliche postoperative Komplikationsraten bei längeren OP-Zeiten fest [39]. Die einzige multizentrische Untersuchung wurde von Yang et al. durchgeführt und umfasste 124 VATS-Pneumonektomien und 235 offene Operationen. Die Outcomes deckten sich mit denen früherer Studien, einschließlich einer Konversionsrate von 19\%, und persistierende Unterschiede bestanden lediglich beim Ausmaß der Lymphknotendissektion, das tendenziell zugunsten der VATS ausfiel [40] (Tab. 5). Zu roboterassistierten Resektionen liegen nur wenig Daten vor. Lediglich 6 Fälle sind beschrieben worden, unter Einsatz verschiedener Konfigurationen. Die Outcomes umfassen 2 Fälle von Konversion und 1 Fall von Komplikation/Mortalität aufgrund einer Pulmonalarterienblutung [41] (Tab. 6).

\section{Technik der roboterassistierten Pneumonektomie}

Die roboterassistierte Pneumonektomie wirft eine Reihe von technischen Problemen auf. Die Exposition der Hauptlungenarterie macht oft die Durchtrennung der Vena pulmonalis superior über einen anterioren Zugang erforderlich. Bei großen hilären Tumoren kann aufgrund der proximalen Ausdehnung eine intraperikardiale Dissektion notwendig werden. Eine frühzeitige Durchtrennung der Lungenvenen kann das Präparat schwer zugänglich machen und die Extraktion erschweren, dadurch können zusätzliche Schritte wie eine stückweise Entnahme erforderlich werden und die Gefahr der Tumorstreuung steigen. Linksseitige Pneumonektomie werden durch die Aorta und das Risiko eines langen Bronchusstumpfs erschwert. Die vollständige Dissektion des Lymphknotengewebes im aortopulmonalen Fenster kann die Retraktion begünstigen und eine
300

Kompass Pneumol 2021;9:292-302 DOI: $10.1159 / 000519794$ 
Tab. 5. Outcomes der videoassistiert-thorakoskopischen Pneumonektomie

\begin{tabular}{|c|c|c|c|c|}
\hline Studie & Art der Studie & Datum & $N$ & Ergebnisse \\
\hline Battoo et al. [38] & Monoz. Kohorte & 2002-2012 & $\begin{array}{l}107 \\
67 \text { VATS } \\
40 \text { offen }\end{array}$ & $\begin{array}{l}\text { Mediane OP-Dauer } 289 \text { (VATS) vs. } 225 \text { Min. (offen) } \\
\text { OP-Mortalität 7,5 (VATS) vs. 5\% (offen); nicht signifikant } \\
\text { Keine Unterschiede bei Komplikationsrate, lokoregionärer Rezidi- } \\
\text { vierung oder WWD } \\
17 \text { Konversionen zu offener OP } \\
\text { Schmerzfreiheit nach } 1 \text { Jahr } 54 \text { (VATS) vs. 19\% (offen) }\end{array}$ \\
\hline Liu et al. [39] & Monoz. Kohorte & $\begin{array}{l}2013-2016 \text { VATS } \\
2010-2013 \text { offen }\end{array}$ & $\begin{array}{l}96 \text { (Parallelisierung 2:1) } \\
32 \text { VATS } \\
64 \text { offen }\end{array}$ & $\begin{array}{l}\text { OP-Mortalität } 0 \text { (VATS) vs. 0\% (offen) } \\
\text { Keine Unterschiede bei Komplikationen, Transfusionen, geschätz- } \\
\text { tem Blutverlust, Drainagedauer oder WWD } \\
\text { OP-Dauer } 187 \text { (VATS) vs. } 146 \text { (offen) } \\
\text { Mittlerer Schmerzscore 2,1 (VATS) vs. 2,6 (offen) }\end{array}$ \\
\hline Pan et al. [30] & Serie & 2014-2015 & 21 & $\begin{array}{l}\text { 30-Tage-Mortalität 4,8\% } \\
\text { R0-Resektionsrate 90,5\% } \\
\text { 4,8\% Konversion zur offenen OP wegen verkalkter Lymphknoten } \\
\text { Komplikationsrate 19\% (2 Pneumonie, } 2 \text { Pyothorax, } 1 \text { Anastomose- } \\
\text { Leckage) } \\
\text { Mittlere OP-Dauer 158 Min. } \\
\text { Durchschnittl. WWD 10,7 Tage }\end{array}$ \\
\hline Yang et al. [40] & $\begin{array}{l}\text { Multiz. } \\
\text { Kohorte }\end{array}$ & 2000-2016 & $\begin{array}{l}359 \\
124 \text { VATS } \\
235 \text { offen }\end{array}$ & $\begin{array}{l}\text { OP-Mortalität } 7 \text { (VATS) vs. 8\% (offen) } \\
\text { Komplikationsrate } 28 \text { (VATS) vs. 28\% (offen) } \\
\text { 5-Jahres-Überleben } 47 \text { (VATS) vs. 33\% (offen); nicht signifikant } \\
\text { 19\% Konversion zur offenen OP }\end{array}$ \\
\hline
\end{tabular}

Tab. 6. Fallserien zur roboterassistierten Pneumonektomie

\begin{tabular}{llll}
\hline Studie & Art der Studie & Ergebnisse \\
\hline Giulianotti et al. [42] & Fallserie & 3 & 2 Konversionen, 1 Todesfall nach PA-Verletzung und -Blutung \\
Spaggiari et al. [43] & Fallserie & 2 & Keine Morbidität oder Komplikationen \\
Rodriguez et al. [44] & Fallbericht & 1 & Keine Morbidität oder Komplikationen \\
\hline
\end{tabular}

weiter proximale Klammernaht ermöglichen. Wir haben dies in den letzten 2 Jahren 8-mal mit Erfolg durchgeführt (Video zu unserer Technik unter https://www.youtube.com/watch?v = P1mrftUCBCg).

Unserer Erfahrung nach ist ein intraperikardiales Vorgehen oft, wenn auch nicht immer, erforderlich, da eine Manschette nicht möglich ist, und kann die Durchtrennung der Lungengefäße erleichtern. Sobald die Dissektion um die Lungengefäße herum weitgehend erfolgt und eine grobe oder multistationäre N2-Erkrankung ausgeschlossen ist, durchtrennen wir in der Regel zuerst die untere Lungenvene, danach die obere Vene und dann die Hauptlungenarterie. Bei einer linksseitigen Pneumonektomie ermöglicht die vollständige Dissektion der subcarinalen und PAFenster-Lymphknoten eine Bronchusdurchtrennung auf Höhe der Carina durch schwarze Handgerät-Klammerung; dieser Schritt wird zuletzt durchgeführt. Wenn möglich, teilen wir das Präparat vor der Extraktion routinemäßig durch, um zwei kleinere Präparate statt eines großen zu bergen und dadurch die Inzisionslänge und den Einsatz von Rippenspreizern zu minimieren. Wenn wir eine solche Teilung vornehmen, erfolgt sie routinemäßig entlang der Fissur.

\section{Diskussion}

Wie weiter oben bereits ausgeführt, ist Lungenkrebs nach wie vor eine Quelle zunehmender Morbidität und Mortalität und die führende krebsbedingte Todesursache in den USA. Dank dem medizinischen Fortschritt können wir heute auch bei fortgeschrittenerem NSCLC ein längeres Überleben bei geringerer Morbidität erreichen als früher. Die chirurgische Resektion war schon immer eine tragende Säule der NSCLC-Therapie, und ihre Rolle in fortgeschrittenen Krankheitsstadien nach einer Induktionstherapie ist noch nicht abschließend etabliert und wird in den kommenden Jahren noch einiger Aufmerksamkeit bedürfen. Das Aufkommen der Roboter-Operationssysteme könnte es möglich machen, diese ebenso wie erweiterte Formen der Resektion, z.B. Pneumonektomien und Manschetten-Lobektomien, so durchzuführen, dass man die Vorzüge einer lokal möglichst begrenzten Resektion auch auf minimalinvasive Weise erzielen kann. Mit der obigen Datenauswertung konnten wir nachweisen, dass eine adäquate Resektion in der Tat möglich ist. In der größten von uns betrachteten Studie zur Operation nach Induktionstherapie wurden vergleichbare R0-Resektionsraten erreicht, und eine Studie mit 20 
Teilnehmern zur MIC-Manschetten-Lobektomie ergab eine R0Resektionsrate von 100\%. Darüber hinaus schienen die kurz- und langfristigen Überlebensraten bei minimalinvasiver und offener Durchführung dieser erweiterten Resektionen statistisch vergleichbar zu sein, wenngleich die Zahl der MIC-Studien sehr begrenzt ist. Auch schienen diese wenigen Studien einen ähnlichen Trend zu niedrigen Raten der Konversion zur Thorakotomie zu zeigen. Mit einem Tiefstwert von 4,5\% lag die Konversionsrate in den ausgewerteten Studien im Durchschnitt bei etwa 20\%. Die häufigsten Ursachen waren Blutungen und/oder stark verkalkte und fibrotische hiläre Lymphknoten, die die Dissektion riskanter machten.

Insgesamt sind wir der Ansicht, dass diese Auswertung vorliegender Daten sowie die Erfahrungen in unserer Einrichtung beweisen, dass die Durchführbarkeit dieser erweiterten Resektionen mit einer Roboter-Plattform gegeben ist. Der wichtigste Punkt ist, dass der Operateur gut vorbereitet ist und weiß, was intraoperativ zu erwarten ist. Unsere Arbeitsgruppe verfolgt eine Strategie, die auf die folgenden grundlegenden Schritte fokussiert ist:

(1) Erlangung hilärer Kontrolle, bevor man mit den Bereichen der kompliziertesten Dissektion fortfährt, insbesondere bei vorausgehender Induktionstherapie;

(2) gründliche Dissektion der LK, um sich ein definitives Bild der Atemwege zu verschaffen, und gute Ausrichtung der Atemwege für die Nahtsetzung; sowie
(3) intraperikardiale hiläre Kontrolle bei MIC-Pneumonektomie. Unserer Überzeugung nach kann ein OP-Team, das diese grundlegenden Schritte zu befolgen vermag und sich dabei stets für einen Umstieg auf Thorakotomie bereithält (mit bereitstehenden Blutprodukten, Thorakotomie-Sieb im OP-Saal, Schwämmen im Thorax für Druckausübung und Zugriff auf kompetente Partner), diese Resektionen auf sichere und wirksame Weise durchführen, um dieses Patientenkollektiv bestmöglich zu versorgen.

\section{Interessenskonflikte}

RC legt Beziehungen zu folgenden Organisationen in der Vergangenheit offen: AstraZeneca, Bard Davol, Bovie Medical Corporation, C-SATS, ConMed, Covidien/Medtronic, Ethicon, Fruit Street Health, Google/Verb Surgical, Intuitive Surgical, KCI/Acelity, Myriad Genetics, Neomend, Pinnacle Biologics, ROLO-7, Tego und TransEnterix.

Die übrigen Autoren erklären, dass bei der Durchführung der Forschungsarbeit keine kommerziellen oder finanziellen Beziehungen bestanden, die als potenzieller Interessenkonflikt ausgelegt werden könnten.

\section{Lizenzangabe}

Joshua A. Scheinerman, Jeffrey Jiang, Stephanie H. Chang, Travis C. Geraci, Robert J. Cerfolio: Extended Robotic Pulmonary Resections. Front Surg. 2021;8:597416 (DOI: 10.3389/fsurg.2021.597416). ${ }^{{ }^{\circ}} 2021$ Die Autoren (Übersetzung; Beiträge der einzelnen Autoren gekürzt), lizensiert unter CC BY 4.0 (https://creativecommons.org/licenses/by/4.0/deed.de).

\section{Literatur}

Die Literatur ist unter www.karger.com/Article/Fulltext/519794 abrufbar. 